\title{
A QFT Based Robust SVC Controller For Improving The Dynamic Stability Of Power Systems
}

\section{P Shrikant Rao and Indraneel Sen}

\begin{abstract}
A novel design technique for an SVC based Power System Damping Controller has been proposed. The controller attempts to place all plant poles within a specified region on the s-plane to guarantee the desired close loop performance. The use of Horowitz's Quantitative Feedback Theory (QFT) permits the design of a "fixed gain controller" which maintains its performance in spite of large variations in the plant parameters during its normal course of operation. The performance of this robust controller has been evaluated on a Single Machine Infinite Bus system equipped with a mid point SVC and the results are shown to be consistent with the expected performance of the stabilizer.
\end{abstract}

\section{Introduction}

Power systems stabilizers (PSS) have been widely used as supplementary controllers to provide extra damping for the synchronous generators in electrical power systems. In spite of the availability of various innovative designs, the fixed gain, lead lag compensation type of stabilizer has been the most popular with the electrical utilities. The increasing utilization of FACTS devices in transmission networks provides an alternative means of designing controllers for damping the low frequency oscillations. Though primarily meant for maintaining a desired voltage profile over the network, the SVCs, with an auxiliary injection of a suitable signal can considerably improve the dynamic stability performance of a power system [1,2].

Continual changes in the system configuration and loading patterns cause corresponding changes in the small signal dynamics of the power system. From the point of view of practical applicability, it is desirable that the controller design is robust to these changes, that is, the controller's performance is maintained over a reasonable range of system and operating conditions. This paper discusses the design of one such SVC based robust controller.

Engineering experience indicate that in a system with a damping factor of about $10 \%$ and $\sigma$ not greater than -0.5 for the troublesome low frequency modes, the oscillations die down within a reasonable time. In terms of the system eigen values, if all the poles of the linearized model of the plant, for all possible operating conditions lie to the left of the D contour shown in Fig. 1, these conditions are met. This property of the closed loop system is henceforth referred to as the 'D-stability' and the system is said to be 'D-stable'. Any controller than can achieve this over a specified range of operating conditions would be robust and would guarantee well damped performance of the controlled system.

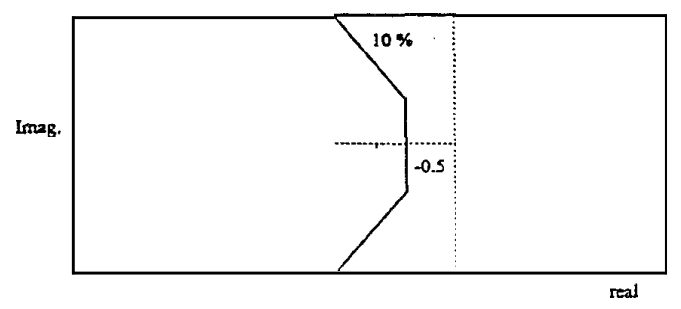

Fig.1 The D-stability Contour

\section{Design of Controller}

The discussion here covers only those aspects of QFT that are relevant to the proposed PSS design and is at a slight deviation from the methodology originally suggested by Horowitz and Sidi [3]. In conventional QFT design, attempts are made to shape the closed loop fiequency response of the system so as to fit it within a pre-specified tolerance band. This is achieved by manipulating the plant templates into certain admissible regions on the Nichols chart by a proper choice of a feedback 
compensator and a prefilter. The procedure followed in this paper is slightly different and focuses on achieving robust stability by manipulating. the plant templates on the Nichols chart and not on shaping the system frequency response.

Consider the configuration shown in Fig.2.

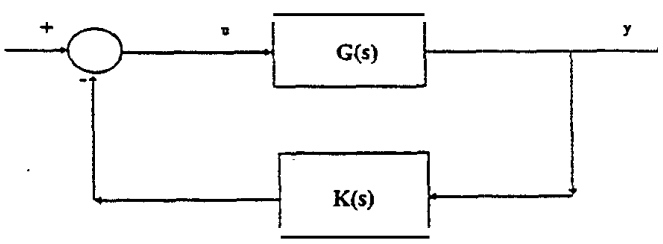

Fig. 2 Variable Plant with Feedback Controller

$\mathrm{G}(\mathrm{s})$ is the plant, which due to the uncertainty in the plant parameters is only known to belong to a set, $\mathscr{G}$. of plants. The value set $G\left(j \omega_{\mathfrak{i}}\right), G \in \mathscr{G}, \omega_{i} \in \Re$, is called the 'plant template' at frequency $\omega_{i}$. A template thus represents the range of variations in the plant response at a particular frequency $\omega_{i}$.

Plant templates can be plotted on the Nichols chart at any desired frequency $\mathbf{a}$, by computing $G(j \omega)$ as $G$ varies over the set $q$ and then manually constructing a boundary around the set of points thus obtained.

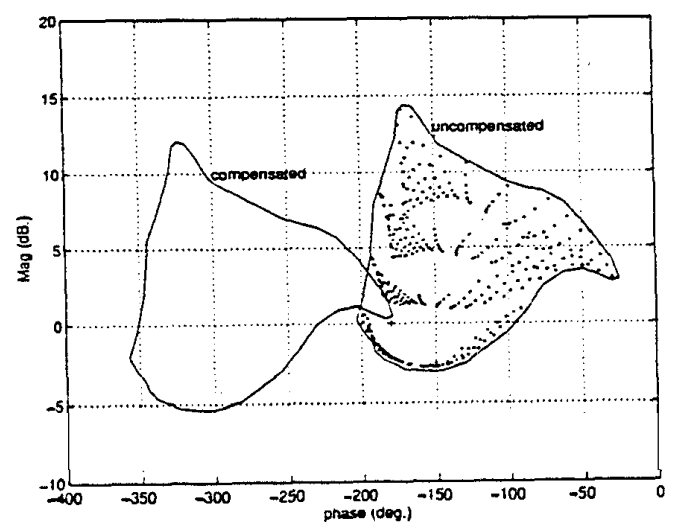

Fig. 3 A Typical Plant Template

Fig. 3 shows a sample template for a particular frequency for the set of plants being considered here. The introduction of the controller $K(s)$, shifts the template at each frequency to a new location on the Nichols chart without altering its shape. This shift depends upon the value of $\mathrm{K}(\mathrm{s})$ at that frequency, Figure 3 shows a typical plant template at its original location as also in the shifted location after the introduction of the controller. Each point on this plot represents a computed value of $G(s)$ as $G$ is varied over the set $\mathcal{G}_{\text {. }}$. The boundary of the template is approximated by straight line segments and is drawn manually by inspection. One plant in $\mathcal{G}$, designated $\mathrm{G}_{0}$, is arbitrarily chosen as the nominal plant.

The closed loop plant will be robustly stable (i.e. stable $\forall \mathrm{G} \in \mathcal{G}$ ), if (a) The set $\mathscr{q}$ is connected (b) The nominal closed loop system is stable and (c) The templates of the compensated plant $(\mathrm{K}(\mathrm{s}) \mathrm{G}(\mathrm{s})$ ) do not contain the $\left(-180^{\circ}, 0 \mathrm{~dB}\right)$ point on the Nichols chart for'd $\omega \in \Re$.

Any controller satisfying the above three conditions, robustly stabilizes the given connected set of plants. These conditions can be extended to cover the D-stability with the modification that (c) should be satisfied for all points on the D-contour instead of the points on the imaginary axis. The plant templates are therefore drawn for the points on the D contour. The templates are then shifted by choosing $\mathrm{K}(\mathrm{s})$, such that none of the templates contains the point $\left(-180^{\circ}, 0 \mathrm{~dB}\right)$. In addition, if the nominal closed loop is D-stable, then robust D stability for the set of plants is achieved. The procedure for designing a robustly $\mathrm{D}$-stabilizing controller for the given set of plants can be summarized as follows.

1.Choose a set of points on the D-contour, for which the robust stability is desired. These points should be closely spaced in the frequency range of interest and in regions where there is a rapid change in the frequency response of the plant.

2. Plot the plant templates for each of the chosen set of points. For this, $G(s)$ is computed as $\mathrm{G}$ is varied over $\mathscr{G}$, in steps small enough to give clearly defined template boundaries. The template boundaries are then drawn manually.

3. Choose a controller for which the nominal closed loop is D-stable and all 
the compensated templates avoid the point $\left(-180^{\circ}, \mathrm{OdB}\right)$ on the Nichols chart. This can be done by interactively shaping the frequency response of the controller as originally suggested by Horowitz $[3,4]$. It can also be done by formulating the above requirements as constraints and then solving the resulting goal attainment or optimization problem.

Some amount of iteration through the procedure might be required before a satisfactory controller is obtained.

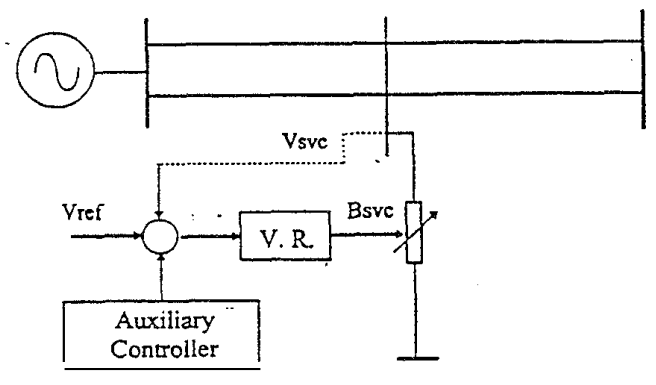

Fig. 4 The Power System Model with SVC

\section{The Power System Model}

A schematic representation of a SMIB system is shown in Fig. 4.

The generator is connected to an infinite bus by a long transmission line which is provided with a mid-point SVC for voltage support. The transmission line represents a double circuit line of $300 \mathrm{~km}$ in length. The generator is fitted with an Automatic Voltage Regulator (AVR) and a static excitation system. Neglecting stator transients and the effects of the damper windings, the generator and SVC can be modeled as a fifth order system with load angle $-\delta$, the rotor speed $\omega$, the internal voltage of the generator $-E_{q}^{\prime}$, the field voltage - $E_{\mathrm{fd}}$ and the SVC susceptance B, as the state variables. A linear time invariant model for this system can be constructed by linearizing the system equations about any given steady state operating condition. The possible variations in operating conditions for this example include variations in the real and reactive powers being supplied by the generator and outages of one of the parallel lines in each of the two sections. For the design example, the real power, $\mathrm{P}$, is assumed to vary from 0.4 to 0.8 p.u. and the reactive power, $Q$, from -0.2 to 0.6. Further, the transmission line reactance of each section is allowed to be either 0.15 or 0.3 p.u. to represent the possible outage of one of the two parallel circuits. Figure 5 shows the plant poles for the above set of operating conditions. As seen, most of the operating conditions do not exhibit desirable pole locations.

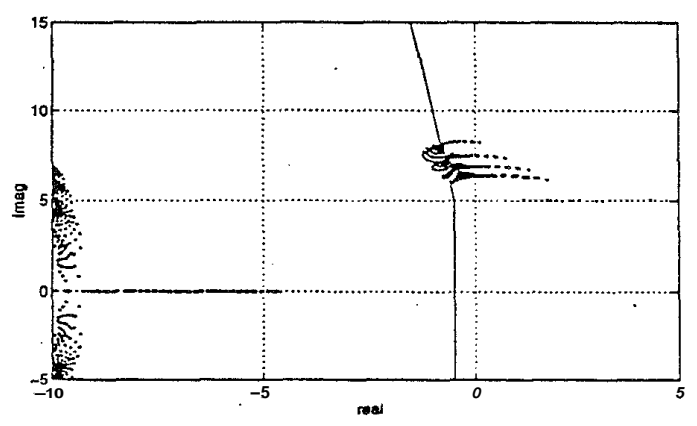

Fig. 5 Open Loop Poles for the Complete Set of Plants

\section{Controller Synthesis}

A controller satisfying the specified requirements of D-stability is obtained by first choosing a particular structure for the controller and then solving an optimization problem to compute the controller parameters.

The problem is formulated by defining a set of constraint functions $g_{i}, i=1, n$, such that $g_{i}>0$ if the $i^{\text {th }}$ compensated template includes the point $\left(-180^{\circ}, 0 \mathrm{~dB}\right)$. To enforce $\mathrm{D}$ stability of the nominal closed loop two additional constraints are required.

$$
\begin{aligned}
& \mathrm{g}_{\mathrm{n}+1}=\max \operatorname{Real}\left(\lambda_{\mathrm{j}}\right)-\sigma \text { and } \\
& \mathrm{g}_{\mathrm{n}+2}=\zeta-\max \left(\operatorname{Damp}\left(\lambda_{\mathrm{j}}\right)\right)
\end{aligned}
$$

where $\lambda_{j}$ are the poles of the nominal plant with controller. Real ( ) represents the real part and Damp ( ) the damping factor of the argument. $\mathrm{g}_{\mathrm{n}+1}, \mathrm{~g}_{\mathrm{n}+2} \leq 0$ ensures that the nominal closed loop is D stable. The problem now reduces to that of finding a controller such that $g, \leq 0 \forall$ i, i.e.,

Find $x$ s.t. $g_{i}(x) \leq 0, i=1,2 \ldots n+2$ 
where $\mathbf{x}$ is a vector of controllerparameters.

\section{Implementation and Results}

The structure chosen for the controller was $\mathrm{K}(\mathrm{s})=\mathrm{K}, .\left(\mathrm{a}, \mathrm{s}^{2}+\mathrm{a}_{2} \mathrm{~s}+1\right) /\left(\mathrm{b}_{1} \mathrm{~s}^{2}+\mathrm{b}_{2} \mathrm{~s}+1\right)$ Where the gain $K$, and the coefficients $a_{1}, a_{2}, b_{1}, b_{2}$ are the tunable parameters. The vector of controller parameters is therefore given by $\mathbf{x}=\left[\mathrm{K}_{\mathrm{s}} \mathbf{a}, \mathrm{a}_{2} \mathrm{~b}_{1} \mathrm{~b},\right]^{\prime}$. A robustly D-stabilizing controller with minimum gain was designed by solving the following optimization problem.

Find $x$ s.t. $K_{s}$ is minimized and $g_{i}$ $\leq 0, \mathrm{i}=1,2 . . \mathrm{n}+2$.

This optimization problem was solved using a program for constrained nonlinear optimization based on sequential quadratic programming available in the Matlab Optimization toolbox [5].

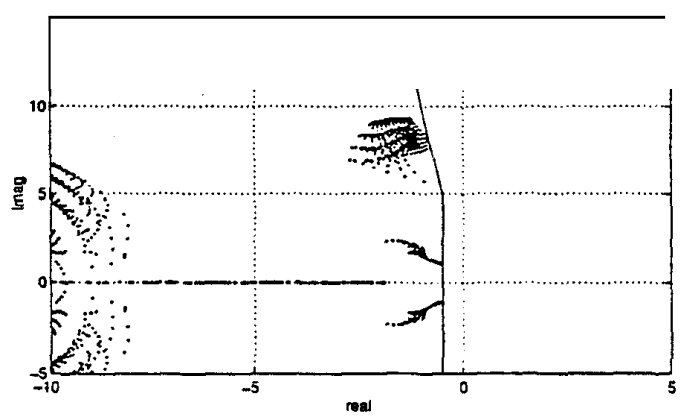

Fig. 6 Closed Loop Poles for the Complete Set of Plants with controller

The parameters of a controller designed to D-stabilize the arbitrarily chosen nominal plant were used as initial guesses during optimization.

The optimization routine yielded a solution for which none of the constraints were violated. Figure 6 shows the closed loop poles of the system fitted with this controller. As seen none of the system poles lie outside the $\mathrm{D}$ contour.

\section{Time Response Plots}

The effectiveness of the proposed controller can be demonstrated by plotting the system response to a small perturbation with and without controller. Fig. 7(a) shows a typical system response to 5\% step disturbance at the AVR input reference.

The system though stable is oscillatory. The performance with the controller is relatively better and meets the design criteria. The system shown in Fig. 7(b) would be unstable without the controller. With controller there is a dramatic improvement in the system performance.

\section{Conclusions:}

An effective method for designing robust damping controllers using SVC devices is presented. The performance requirement for the closed loop plant has been defined in terms of placement of dominant poles on the s-plane. It has been shown that the proposed stabilizer meets this design specification over a wide range of operating and system conditions.

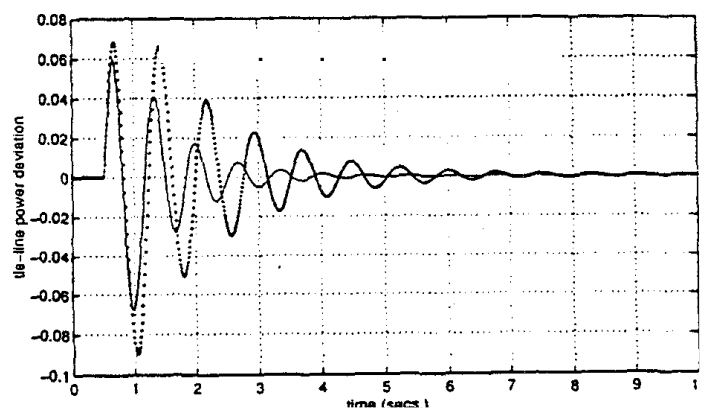

(a) $\mathrm{P}=0.8 \quad \mathrm{O}=0.4 \quad \mathrm{Xe}=0.3$

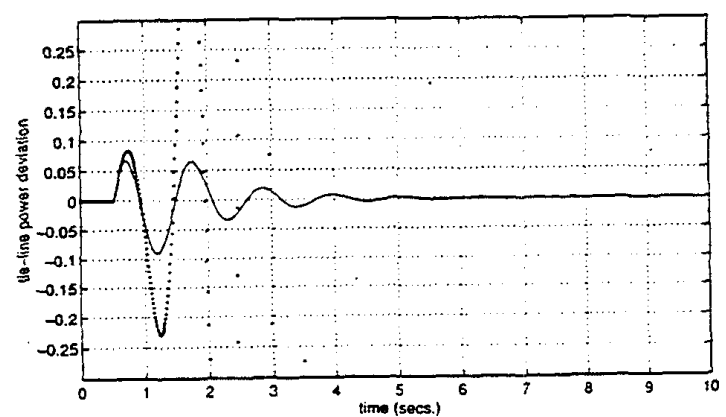

(b) $\mathbf{P}=0.8 \quad \mathrm{Q}=-0.2 \quad \mathrm{Xe}=0.6$

Fig. 7 System Response with and without controller to $5 \%$ Step Disturbance 


\section{Appendix}

System Data:

The generator has been modeled as a third order system and 'the AVR and SVC voltage regulator as first order ( single time constant) systems.

Generator: $X_{d}=2.0, X_{a}=1.7, X_{d}^{\prime}=0.244$

$\cdot \mathrm{T}_{\mathrm{do}}{ }^{\prime}=4.18, \mathrm{H}=3.25, \mathrm{w}_{\mathrm{b}}=314.15$

AVR : $\mathrm{K}_{\mathrm{a}}=50.0, \mathrm{~T}_{\mathrm{a}}=0.05$

$\mathrm{SVC}: \mathrm{K}_{\mathrm{b}}=3.33, \mathrm{~T}_{\mathrm{b}}=0.02$

\section{References}

1.E.V.Larsen, J.J.SanchezGasca and J.H.Chow, 'Concepts for design of FACTS controllers to damp power swings', IEEE PWRS, v.10(2), 1995, p.948.

2.A.E.Hammad 'analysis of power system stability enhancement by SVC', IEEE PWRS v.1:(4), 1986, p.222

3.1. Horowitz, M. Sidi, 'Synthesis of feedback systems with large plant ignorance for prescribed time domain tolerances.', Intl. J1. of Control, Vol.16:(2), 1972, p.287

4.I. Horowitz, 'Survey of Quantitative Feedback Theory', Intl. J1. of Control, Vol. 53:(2) 1991, p.255.

5. Andrew Grace, 'Matlab Optimization Toolbox - Users' Guide' ,Mathworks Inc. 